\title{
A combined laboratory and numerical study of heat transport by baroclinic eddies and axisymmetric flows
}

\author{
By PETER L. READ $\dagger$ \\ Department of Physics, University of Oxford, UK
}

(Received 31 May 2002 and in revised form 17 March 2003)

The effective contribution to the lateral heat transport in a rotating differentially heated annulus attributable to fully developed baroclinic eddies is determined by the combined use of laboratory measurements and numerical simulations. The total heat transport is determined in the laboratory by real-time calorimetry to a precision of around $\pm 2.5 \%$ over a wide range of parameters sampling a wide cross-section of the regular baroclinic wave regime accessible in the rotating annulus up to the transition to geostrophic turbulence. High-resolution numerical simulations of steady axisymmetric flow in the rotating annulus were carried out under comparable parametric conditions to the laboratory experiments, to determine the contribution to total heat transport due to the axisymmetric boundary-layer circulation in the system. The difference between the Nusselt or Péclet numbers determined in these two ways enables the heat transport attributable to the presence of the baroclinic eddies to be determined unambiguously. The variation of the resulting excess Péclet number with external parameters appears to be consistent with predictions from a weakly nonlinear model of baroclinic instability within the regular baroclinic wave regime, at least for weak-moderate supercriticality, whereas at higher rotation rates a parametrization based on the linear instability approach of Green (1970) may be more appropriate. This approach seems to offer an accurate and incisive means of evaluating schemes proposed to parametrize the transport properties of baroclinic eddies in a variety of models used in geophysical and engineering applications.

\section{Introduction}

The problem of how to represent the transport of heat and other quantities by unresolved processes in numerical models is ubiquitous in a variety of disciplines. In the atmospheric sciences, there is a long history of attempts to quantify the effects of large- and small-scale non-axisymmetric eddies on the zonally symmetric structure and circulation (e.g. Green 1970; Stone 1972, 1978; Pfeffer \& Barcilon 1978; Taylor 1980), although the urgency of such a task has become less acute in recent years as the computational cost of eddy-resolving atmospheric models has reduced (apart, perhaps, from their use in problems of very long-term climate change). In oceanography, there remains an urgent need to develop an accurate means of parametrizing the effects of energetic baroclinic eddies within coarse-resolution ocean models. Despite

$\dagger$ Present address: Atmospheric, Oceanic \& Planetary Physics, Clarendon Laboratory, Parks Road, Oxford, OX1 3PU, UK. 
major advances in recent years in available computing power and the capabilities of numerical models to simulate many features of the general circulation of the oceans, the resolution required by such models to resolve adequately the structure and behaviour of even the most energetic baroclinic eddies (around $0.1^{\circ}$ in latitude and longitude according to Smith et al. 2000) remains well beyond that practicable for climate studies. For the foreseeable future, therefore, virtually all such models used for studies of climate and global change will need to parametrize the transfer properties of the dominant energy-containing eddies in the oceans. The practical need to improve the parametric representation of geostrophic eddies for the study of the oceans on climatic timescales thus remains as urgent as ever.

Such problems are not unique to the geophysical world, but similar issues also arise in the design and operation of large-scale rotating machinery in which free thermal convection regimes may lead to significant heat transport which is difficult to predict without the use of expensive computational fluid dynamics (CFD) codes (e.g. Owen $\&$ Rogers 1995). In the present paper, we concentrate on the problem of determining and quantifying the integrated transport of heat due to baroclinic eddies, which has its main application in the fields of oceanography and climate modelling, although the methodology outlined herein may also be useful in other disciplines.

\subsection{Approaches to parametrization}

Despite the widely acknowledged importance of the geostrophic eddy field in the ocean, its representation in large-scale ocean models is often rudimentary. A variety of approaches to the problem of parametrizing baroclinic transports have been adopted over the past 30 years, ranging from simple ideas based on linear and weakly nonlinear baroclinic instability models (e.g. Green 1970; Stone 1972, 1978; Pfeffer \& Barcilon 1978) to more sophisticated treatments using the transformed Eulerian mean formalism and the theory of eddy-mean flow interaction (Gent \& McWilliams 1990; Gent et al. 1995; Visbeck et al. 1997; Treguier, Held \& Larichev 1997).

Current parametrizations generally assume either that conserved tracers such as heat, salinity or passive tracer (whose concentration is denoted for the time being by $s$ ) are diffused down the large-scale gradient, so that eddy fluxes are related to mean quantities either via equations of the form

$$
\overline{v^{\prime} s^{\prime}}=-\mathscr{K} \nabla \bar{s},
$$

where the overbar denotes an averaging operator in space and/or time and the prime denotes departures from that mean, or that they are related to a spatially averaged 'Transformed Eulerian Mean' circulation which is, in turn, derived from the form of the horizontal eddy buoyancy flux $\overline{v^{\prime} b^{\prime}}$. $\mathscr{K}$ in the most general case in (1.1) is a (tensor) transfer coefficient (e.g. Plumb \& Mahlman 1987) that is assumed to depend, crucially, on the local, averaged quantities. This representation raises many questions, among the most important being: Is such a form physically reasonable? What value should $\mathscr{K}$ have? Should $\mathscr{K}$ vary in space and time?

It has long been recognized that $\mathscr{K}$ is often strongly anisotropic. Thus the (nearly) horizontal transfer due to baroclinic eddies vastly exceeds the vertical mixing due to internal wave breaking (see Large, McWilliams \& Doney 1994 for a review of this topic which is not considered further here). Work based on analyses of baroclinic instability by Green (1970) and Stone (1972) suggested that vigorous eddy transfer should occur along surfaces inclined at half the average isopycnal slope. Recently, several studies have advocated a strictly adiabatic closure (Redi 1982; 
Gent \& McWilliams 1990; Gent et al. 1995). Moreover, a clear distinction between the symmetric (diffusive) and anti-symmetric (advective) parts of the $\mathscr{K}$-tensor has been made (see also Plumb \& Mahlman 1987; Griffies 1998; Greatbatch 1998). Current theory focuses on constraints for $\mathscr{K}$ based on physically realizable boundary conditions (Treguier et al. 1997), linear instability analysis (Killworth 1997) and the link to large-scale potential vorticity (Greatbatch 1998; Marshall, Williams \& Lee 1999). The latter also permits a partial treatment of Reynolds stresses for coarseresolution ocean general circulation models, a topic that has, otherwise, received very little attention. Nevertheless, despite this intense activity and the pressing need to resolve this issue, there is no widespread support for (1.1) or an accurate theory for $\mathscr{K}$.

\subsection{Testing closure theory}

Large-scale ocean modellers using these recent parametrizations have often found significant improvements in their solutions (Danabasoglu, McWilliams \& Gent 1994; Robitaille \& Weaver 1995; Wright 1997) in comparison with observational constraints. However, this success clearly does not constitute a comprehensive evaluation of the theories. The most stringent tests to date typically determine the dependence of $\mathscr{K}$ (determined via direct computation of eddy variances or local fluxes) on the largescale fields from heuristic theoretical arguments or empirically from observations or eddy-resolving models.

Several groups in recent years have attempted to develop closures for parametrizations based on the above approaches using results from high-resolution model simulations (Visbeck et al. 1997; Haine \& Marshall 1998; Held \& Larichev 1996; Larichev \& Held 1995; Pavan \& Held 1996). These model studies seek to resolve the detailed behaviour of individual eddies in a variety of idealized circumstances, from which the average transport properties of the eddies can be obtained diagnostically. Visbeck et al. (1997), for example, make use of a limited range of idealized eddyresolving numerical simulations to test their scheme, and this continues to be the most common overall approach to testing the closure schemes. Such approaches are themselves subject to substantial uncertainties, however, due to the severe limitations of the high-resolution models in terms of resolution with respect to the smallest eddies, ranges of accessible parameters, approximations and systematic errors due to numerical schemes. Further, this kind of study has generally attempted only to diagnose the Eulerian eddy fluxes from the resolved model fields, rather than determining the total integrated transport by the flow. Since the pioneering work of Andrews \& McIntyre (1978), it has been appreciated that the total transport of a material tracer is determined not only by the direct eddy correlation $\overline{v^{\prime} s^{\prime}}$, but also via the mean 'Stokes drift'. Furthermore, the distinction between 'eddy fluxes' and 'Stokes drift' is effectively an artifact of taking zonal averages along arbitrary coordinate surfaces, rather than taking (more rigorously correct) Lagrangian means. We note in passing that the task of obtaining the Lagrangian mean transport directly from numerical simulations alone is not straightforward. Moreover, the Nusselt number (ratio of total heat transport to explicit diffusive conduction) is notoriously difficult to simulate accurately in numerical models.

Laboratory systems offer an alternative approach, which does not suffer from the problems of calibration or (usually) the need to invoke controversial parametrizations of unresolved components of the flow. Visbeck, Marshall \& Jones (1996) made use of a range of laboratory experiments on baroclinically unstable convective chimneys 
as part of the validation of their study, with particular reference to the properties of deep convection in the ocean. More relevant to the present context, Pfeffer, Buzyna \& Kung (1980) carried out a major study closely comparable to the work reported herein, though only obtained measurements of Eulerian heat fluxes $\overline{v^{\prime} T^{\prime}}$ and temperature variances $\overline{T^{\prime 2}}$ in a rotating thermally driven annulus, via a combination of thermal and velocity measurements obtained with a dense network of in situ thermistor probes distributed throughout the flow. Despite this, they covered many of the classical regular wave regimes of the rotating annulus and the transition region of geostrophic turbulence, though only on a nominal $f$-plane. There were also some concerns over the extent to which their dense network of probes disturbed the flow, and their results were only compared in any detail with their own weakly nonlinear baroclinic parametrizations (Pfeffer \& Barcilon 1978).

\subsection{Laboratory measurements of heat transport}

In the present paper, therefore, we propose a complementary approach to the quantitative evaluation of parametrization schemes and closures. We make use of direct determinations of the integrated transport of heat by baroclinic eddies in a series of laboratory experiments, combined with the use of a numerical model with a proven capability of accurately simulating the flow in such experiments to a relatively high degree of precision. Previous experiments in a thermally driven rotating fluid annulus have shown that the total heat transport in a baroclinically unstable flow can be determined experimentally (via various forms of real-time calorimetry; e.g. see Hignett 1982; Hignett et al. 1985) to an absolute accuracy better than $\pm 2.5 \%$, and to a relative precision considerably better than this. The difference in total heat transport between the measured value affected by the fully three-dimensional flow and that obtained in a two-dimensional axisymmetric flow under otherwise identical conditions can then be attributed to the influence of the eddies themselves independent of the distinction between averaged eddy correlations and 'Stokes drift'.

Furthermore, because laboratory experiments deal with realizations of baroclinic flows in a real physical fluid, such studies are not subject to the potential systematic errors due to numerical approximations in (even so-called eddy-resolving) numerical models. Thus, provided that parameter regimes and flow configurations which are dynamically similar and relevant to the oceans can be realized in the laboratory, such studies can provide very valuable information on the transport properties of baroclinic flows which is highly complementary to that available by more conventional means.

The present paper is therefore structured as follows. Section 2 describes the laboratory system and available measurements in further detail, outlining the range of regimes covered. Section 3 presents the corresponding numerical simulations, and describes the ways in which these simulations are used to determine the axisymmetric contribution to total heat transport. The main results on the dependence of heat transport on various external parameters are presented in $\S 4$, and the context and significance of the results are discussed, together with concluding remarks and suggestions for further work, in $\S 5$.

\section{Apparatus and measurements}

The experimental system consisted of a differentially heated rotating annulus, in which a fluid (typically a water-glycerol mixture of approximately 17\% glycerol 


\begin{tabular}{|c|c|c|c|}
\hline Parameter & Symbol & Present range & Units \\
\hline $\begin{array}{l}\text { Rotation rate } \\
\text { Temperature difference }\end{array}$ & $\begin{array}{c}\Omega \\
\Delta \mathrm{T}\end{array}$ & $\begin{array}{c}0-4 \\
4\end{array}$ & $\begin{array}{l}\operatorname{rad~s}^{-1} \\
\mathrm{~K}\end{array}$ \\
\hline $\begin{array}{l}\text { Fluid properties: } \\
\text { Density } \\
\text { Thermal expansion } \\
\text { Kinematic viscosity } \\
\text { Thermal diffusivity } \\
\text { Specific Heat }\end{array}$ & $\begin{array}{l}\rho_{0} \\
\alpha \\
v \\
\kappa \\
\mathrm{c}\end{array}$ & $\begin{array}{c}1043-1053 \\
3.3 \times 10^{-4} \\
2.1 \times 10^{-6} \\
1.3 \times 10^{-7} \\
3764\end{array}$ & $\begin{array}{l}\mathrm{kg} \mathrm{m}^{-3} \\
\mathrm{~K}^{-1} \\
\mathrm{~m}^{2} \mathrm{~s}^{-1} \\
\mathrm{~m}^{2} \mathrm{~s}^{-1} \\
\mathrm{~J} \mathrm{~kg}^{-1} \mathrm{~K}^{-1}\end{array}$ \\
\hline $\begin{array}{l}\text { Channel geometry: } \\
\text { Gap width } \\
\text { Mean fluid depth }\end{array}$ & $\begin{array}{c}L=b-a \\
D\end{array}$ & $\begin{array}{c}0.055 \\
0.14\end{array}$ & $\begin{array}{l}\mathrm{m} \\
\mathrm{m}\end{array}$ \\
\hline $\begin{array}{l}\text { Non-dimensional: } \\
\text { Ekman number (equation (2.4)) } \\
\text { Thermal Rossby number (equation (4.1)) } \\
\text { Prandtl number }\end{array}$ & $\begin{array}{c}E \\
\Theta \\
\sigma(=v / \kappa)\end{array}$ & $\begin{array}{c}10^{-5}-10^{-4} \\
0.01-2 \\
16.8\end{array}$ & \\
\hline
\end{tabular}

by volume, with properties summarized in table 1) was contained between two upright coaxial brass cylinders of radii $a=2.5 \mathrm{~cm}$ and $b=8.0 \mathrm{~cm}$ respectively, and rigid thermally insulating (Perspex), horizontal endwalls at $z=0$ and $z=14.0 \mathrm{~cm}$. The annulus was rotated about its vertical axis of symmetry at angular velocity $\Omega$. The apparatus was designed to make accurate measurements of absolute heat transport through the inner sidewall, by real-time calorimetry, and is described in detail by Hignett $(1982,1985)$ and Hignett et al. (1985). From measurements of (i) the flow rate of coolant water through the inner cylinder and (ii) the small difference in temperature between the inlet and outlet of the coolant system, the total heat flux passing through the sidewall could be determined. Leakage of heat into the system from the apparatus environment was taken into account by carrying out a careful series of null experiments, in which the working fluid was replaced by filling the annular gap with a piece of solid expanded-polystyrene insulator. By this means, the total heat transport through the inner sidewall could be determined to an absolute precision of approximately $\pm 2.5 \%$.

The Nusselt number was defined by non-dimensionalizing the total heat transport $H$ by that due to thermal conduction through a solid of the same thermal conductivity as the working fluid, thus

$$
N u=\frac{H \ln (b / a)}{2 \pi \kappa D \rho c\left(T_{b}-T_{a}\right)},
$$

where $\kappa$ is the thermal diffusivity of the fluid, $\rho$ its density and $c$ its specific heat capacity. The series of measurements include those discussed by Hignett $(1982,1985)$ and others taken at around the same time which cover the regular and incipient irregular wave regimes. More recently, a new calibration of the null state (without the working fluid in place) was carried out (W. G. Früh, personal communication), which resulted in a correction to Hignett's original Nusselt numbers by a factor of 1.1165. This brings the wave results into consistent agreement with the earlier series of 


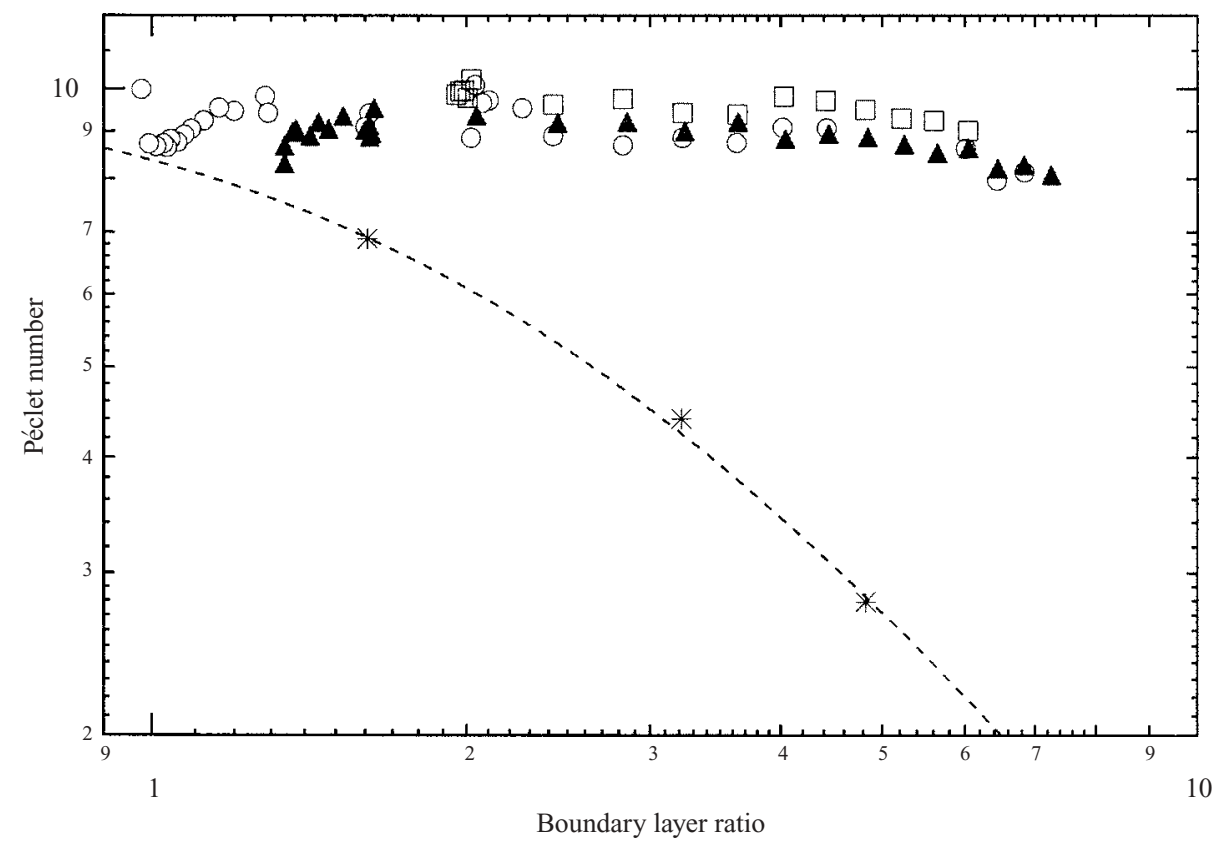

FIGURE 1. Variation of measured total heat transport in a rotating differentially heated annulus with boundary layer parameter $\mathscr{P}$, over the range where baroclinic waves are found to occur. Time-averaged heat transport is shown as a Péclet number for each wavenumber $m$; $\bigcirc, m=2 ; \triangle, m=3 ; \square, m=4$. The dashed line shows the variation of $P e$ computed in numerical simulations of axisymmetric flow under the same conditions as the laboratory experiments, obtained via a fit to computations shown here as asterisks.

axisymmetric measurements of Hignett (1982). The resulting measurements represent the total heat transport averaged over 1-2 hours in each case.

The resulting measurements are listed for completeness in tables 3, 4 and 5, which are not printed here but are available on request from the author or the Journal of Fluid Mechanics Editorial Office, Cambridge. The results are also shown in figure 1 as a series of points plotted as Péclet number $(P e=N u-1)$ against the parameter $\mathscr{P}$, defined as the squared ratio of the boundary layer thicknesses of the sidewall thermal boundary layer and the Ekman layer (Hignett, Ibbetson \& Killworth 1981; Hignett 1982; Read 1986):

$$
\mathscr{P}=R a^{-1 / 2} E^{-1} \epsilon^{-3 / 2},
$$

where $R a$ is the Rayleigh number,

$$
R a=\frac{g \alpha \Delta T L^{3}}{\kappa \nu},
$$

$E$ is the Ekman number

$$
E=\frac{v}{2 \Omega D^{2}},
$$

and $\epsilon$ is the meridional aspect ratio

$$
\epsilon=\frac{D}{L} .
$$


Also, $g$ is the acceleration due to gravity, $\alpha$ is the thermal expansion coefficient, $L=b-a, D$ is the annulus height and $v$ is the kinematic viscosity. With this definition, $\mathscr{P}$ has the useful property of being directly proportional to $\Omega$. Also, as noted by Hignett et al. (1981), Hignett (1982) and Read (1986), the parameter $\mathscr{P}$ provides a clear means of establishing the quantitative role of Ekman layers in heat transport since, when $\mathscr{P}$ is much less than unity, the Ekman layers can accommodate any meridional flow forced in the buoyancy-dominated $R a^{1 / 4}$ sidewall thermal boundary layers. Where $\mathscr{P}>1$, however, Ekman layers become significantly narrower than the thermal boundary layers and inhibit radial advective heat transport. It is also at around $\mathscr{P} \simeq 1$ that baroclinic instability tends to occur so that the baroclinic wave regime is found for $\mathscr{P}>1$.

A major issue in the baroclinic wave regime, however, is that the total heat transport includes contributions from both the baroclinic eddies themselves and the axisymmetric boundary-layer circulation. In order to isolate the baroclinic eddy contribution, therefore, it is necessary to separate this from the contribution due to the boundary-layer-dominated circulation in the absence of baroclinic eddies. This cannot be done readily from direct experiments, since baroclinic instability always occurs in the parameter region $\mathscr{P}>1$ (unless special precautions are taken, e.g. using sloping boundaries, see Hignett 1982). However, it is possible to compute the boundary-layerdominated axisymmetric contribution to heat transport from a numerical simulation, and this is described in the next section.

\section{Numerical model and axisymmetric heat transport}

The numerical model used to compute the axisymmetric boundary-layer heat transport is the two-dimensional axisymmetric version of the finite-difference timedependent Boussinesq model described by Hignett et al. (1985) and as model $E$ of Read, Thomas \& Risch (2000). This model solves the full non-hydrostatic NavierStokes equations for a Boussinesq fluid in cylindrical annular geometry, subject to specified boundary conditions at the side- and endwalls. Conservative Eulerian finitedifference schemes are used to ensure accurate global conservation of temperature (potential energy) and kinetic energy.

For the present purpose, the model was run using a grid resolution of up to $96 \times$ 96 points in $(r, z)$, with a stretched mesh in both $r$ and $z$ using a hyperbolic-tangent stretch on lengthscales corresponding to the $R a^{-1 / 4}$ thermal boundary layer and Ekman layer thicknesses. The boundary conditions corresponded to rigid non-slip isothermal boundaries at the sidewalls, and thermally insulating conditions at the horizontal endwalls. Under these conditions, the model has been shown (Read et al. 2000) to simulate the total heat transport in the axisymmetric regime to an absolute accuracy at least as good as that of the experimental measurements themselves (around $\pm 2.5 \%$ ). For each case, the model was run for a minimum of $5000 \mathrm{~s}$ (with a typical timestep of $0.05 \mathrm{~s}$ ), by which simulated time the flow had equilibrated to a nearly steady state and residual variations in $N u$ were within $1 \%$ of the final mean value.

Model runs were carried out over a wide range of $\Omega$, covering regimes which include both low-rotation axisymmetric flows and those where the fully three-dimensional flow would become baroclinically unstable. The resulting simulations thus represent valid steady-state axisymmetric solutions to the Navier-Stokes equations under the same conditions as the fully developed baroclinic wave flows in the laboratory. Some typical fields from a representative sample of simulations covering the very 
(a)

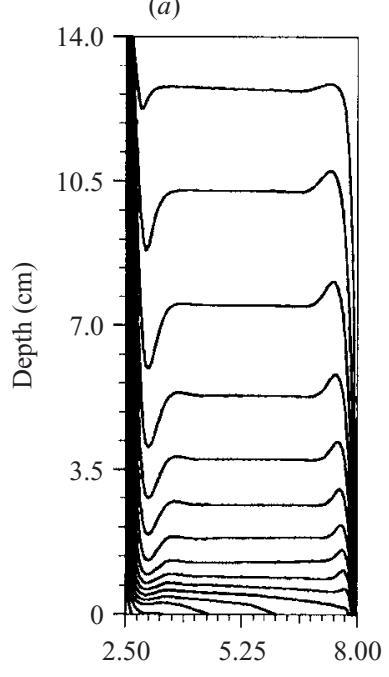

(d)

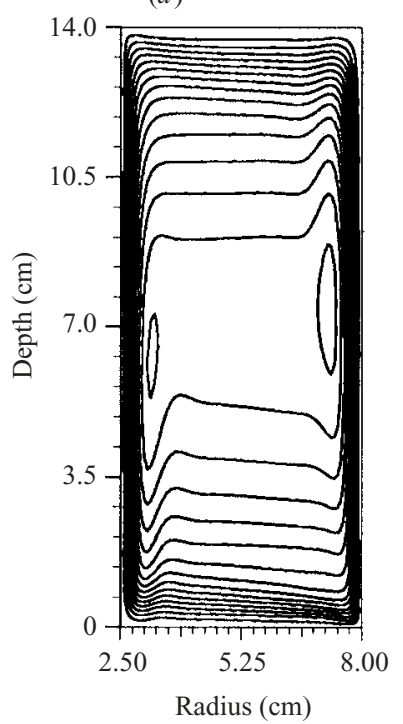

(b)

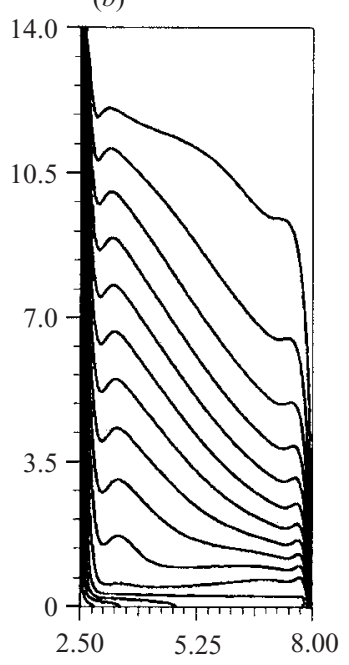

(e)

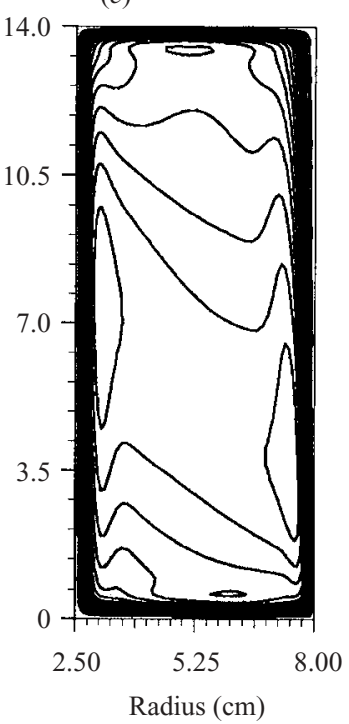

(c)

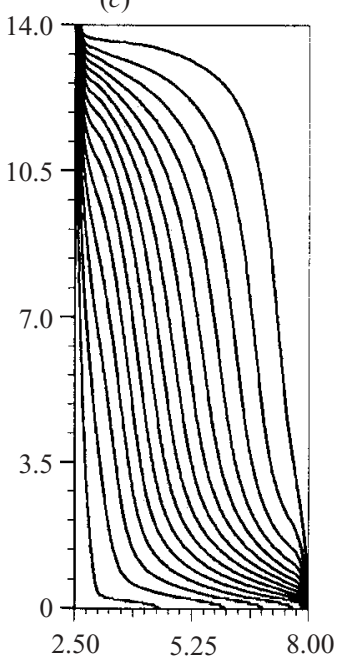

FIGURE 2. Maps in $(r, z)$ of $(a-c)$ temperature and $(d-f)$ meridional streamfunction in numerical simulations of flow in a differentially heated rotating annulus as discussed in the text. Simulations were carried out at three rotation rates, typical of very low rotation rates $\left(\Omega=0.01 \mathrm{rad} \mathrm{s}^{-1}\right)(a, d)$; intermediate rotation rates $\left(\Omega=1.0 \mathrm{rad} \mathrm{s}^{-1}\right)(b, e)$; and the high rotation regime $\left(\Omega=3.0 \mathrm{rad} \mathrm{s}^{-1}\right)(c, f)$. Contour intervals are $0.25 \mathrm{~K}$ for $(a-c)$, and (d) $0.025 \mathrm{~cm}^{2} \mathrm{~s}^{-1},(e) 0.01 \mathrm{~cm}^{2} \mathrm{~s}^{-1}$ and $(f) 0.005 \mathrm{~cm}^{2} \mathrm{~s}^{-1}$.

low-rotation, intermediate-rotation and high-rotation states are illustrated in figure 2. Figure 2(a-c) presents the temperature cross-sections for these cases, and clearly show the transition from a highly relaxed temperature field at low rotation rates, with near-horizontal isotherms in the interior, to near-vertical isotherms at high rotation, reflecting the effect of the thinning of the Ekman layers in suppressing horizontal heat 


\begin{tabular}{ccccc}
\hline$\Omega\left(\mathrm{rad} \mathrm{s}^{-1}\right)$ & $E$ & $P$ & $\Theta$ & $N u$ \\
0.01 & $5.408 \times 10^{-3}$ & 0.0161 & $6.19 \times 10^{3}$ & 11.26 \\
0.03 & $1.803 \times 10^{-3}$ & 0.0482 & $6.88 \times 10^{2}$ & 11.25 \\
0.05 & $1.082 \times 10^{-3}$ & 0.0804 & $2.48 \times 10^{2}$ & 11.23 \\
0.1 & $5.408 \times 10^{-4}$ & 0.1608 & 61.9 & 11.16 \\
0.2 & $2.704 \times 10^{-4}$ & 0.3216 & 15.5 & 11.09 \\
0.3 & $1.803 \times 10^{-4}$ & 0.4825 & 6.88 & 10.94 \\
0.5 & $1.082 \times 10^{-4}$ & 0.8041 & 2.48 & 10.07 \\
1.0 & $5.41 \times 10^{-5}$ & 1.608 & 0.619 & 7.89 \\
2.0 & $2.70 \times 10^{-5}$ & 3.216 & 0.155 & 5.4 \\
3.0 & $1.80 \times 10^{-5}$ & 4.825 & 0.0688 & 3.79 \\
TABLE 2. Results of axisymmetric numerical simulations of heat transport in the rotating \\
\multicolumn{7}{c}{ annulus described in the text at $R a=8.06 \times 10^{6}$. } \\
\hline \multicolumn{7}{c}{}
\end{tabular}

transport. Figure $2(d-f)$ shows the corresponding meridional streamfunction fields, where the streamfunction $\chi_{a x}$ is defined by

$$
\begin{aligned}
& w=-\frac{1}{r} \frac{\partial \chi_{a x}}{\partial r}, \\
& u=\frac{1}{r} \frac{\partial \chi_{a x}}{\partial z},
\end{aligned}
$$

so that meridional flow circulates anticlockwise around local maxima in $\chi_{a x}$. The reduction in the horizontal Ekman boundary thickness with increasing $\Omega$ is clearly seen in figure $2(d-f)$. The total heat transport in the simulations is listed in table 2 and represents the total (advective plus conductive) transport due only to the axisymmetric boundary layer flow. Under conditions where the flow is baroclinically unstable in the laboratory, the numerically computed Nusselt number therefore comprises the contribution to the fully three-dimensional total heat transport which needs to be subtracted in order to isolate the contribution due solely to the baroclinic eddies.

While it would have been possible in principle to carry out a model run for every case for which experimental measurements were available, such an exercise would have been computationally expensive and cumbersome, and so an alternative and more flexible approach was used here which made use of the scaling theory developed by Hignett et al. (1981), Hignett (1982) and Read (1986, 1992). As mentioned above, according to this analysis, the axisymmetric advective heat transport depends primarily on the boundary layer parameter $\mathscr{P}$ such that, at small values of $\mathscr{P}$, the heat transport due to advection (measured by the dimensionless Péclet number $P e$ ) is then independent of $\Omega$ and $P e$ is of order $R a^{1 / 4}$. At higher rotation rates, however, where $\mathscr{P}>1, P e$ asymptotically becomes inversely proportional to $\Omega^{3 / 2}$ such that $P e \sim$ $R a^{1 / 4} \mathscr{P}^{-3 / 2}$. Based on a limited series of numerical simulations, therefore, spanning the full range of $\mathscr{P}$ covered by the laboratory measurements, and with aspect ratios and $R a$ equal to the mean values of the experiments themselves, we have fitted an empirical function of the form

$$
P e_{n}=\frac{A}{\left(\mathscr{P}^{3 / 2}+B\right)},
$$




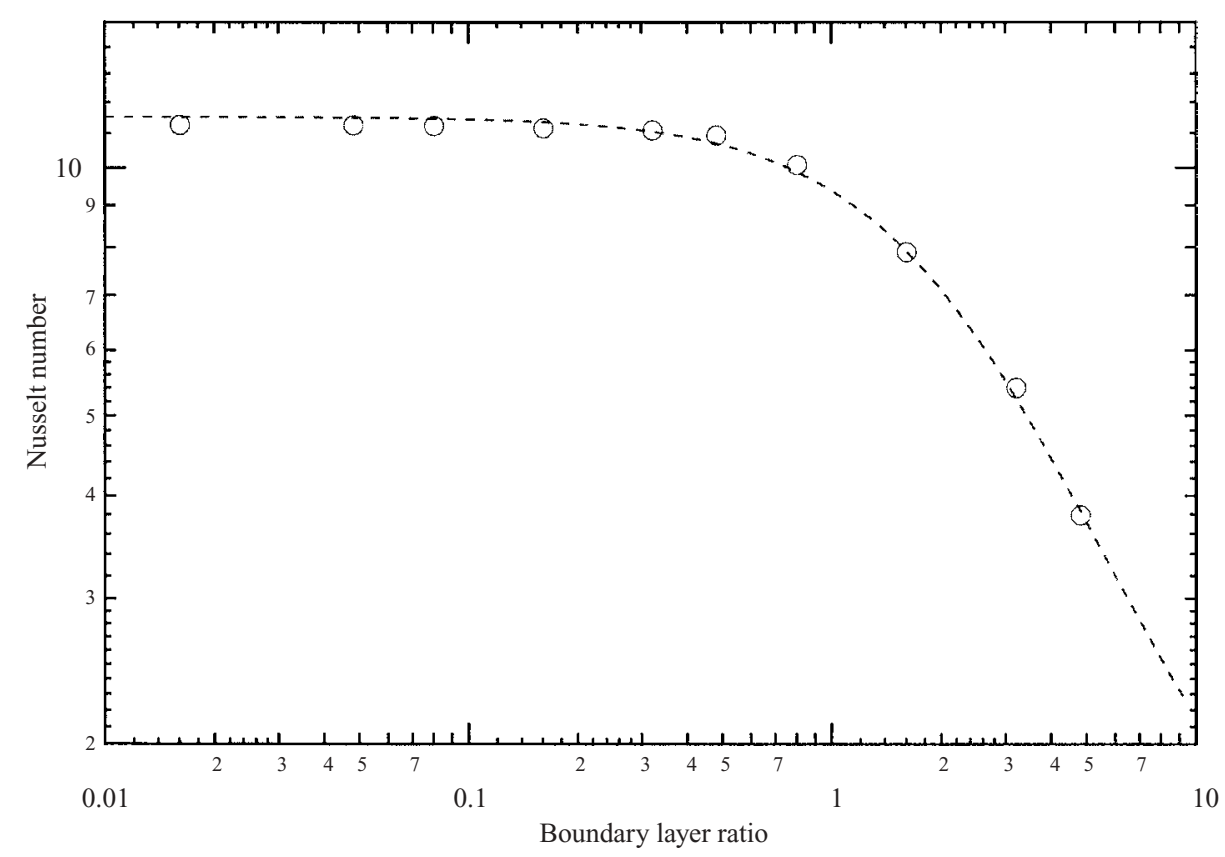

FIGURE 3. Variation of total heat transport in axisymmetric numerical simulations of flow in a rotating differentially heated annulus with boundary layer parameter $\mathscr{P}$, covering the full range of rotational regimes. The time-averaged heat transport for each simulation is shown as an open circle representing the Nusselt number. The dashed line is a least-squares fit to the individual simulations with a function of the form $N u=1+A /\left(P^{3 / 2}+B\right)$, illustrating the transition from the low-rotation regime $(N u$ independent of $\mathscr{P})$ to the high-rotation state $\left(P e_{n}=\mathrm{O}\left(\mathscr{P}^{-3 / 2}\right)\right.$.)

to the Péclet numbers $(P e=N u-1)$ derived from the simulations, obtaining the values $A=40.98 \pm 0.63$ and $B=3.894 \pm 0.085$ as constants. The resulting fit for $N u$ is shown in figure 3 as a dashed line, together with the values of $N u$ from the original simulations, and is clearly seen as a reasonably good fit (to within $1-2 \%$ of the nonrotating value of $N u$ ) over the entire range of $\mathscr{P}$ of interest. For the purposes of this study, therefore, we take the relationship given in (3.3) to represent the dependence of the axisymmetric heat transport to within the precision of the experimental measurements.

\section{Results: baroclinic eddy heat transport}

From the predicted axisymmetric heat transport in $\S 3$, the axisymmetric contribution to the total heat transport in the fully three-dimensional flow in the laboratory can be isolated from the measurements introduced in $\S 2$. The resulting difference in heat transport must therefore represent the integrated transport attributable to the baroclinic eddies themselves (in the form of a combination of both a direct eddy flux and a modification of the zonally symmetric meridional transport due to the action of the eddies). From a comparison of the measured Nusselt numbers and the numerically predicted axisymmetric Nusselt numbers shown in figure 1, it is clear that the difference $P e_{x s}=N u_{\text {total }}-N u_{a x}$, and therefore the heat transport attributable to the eddies, increases steadily with $\mathscr{P}$ from a 'critical' value of around $\mathscr{P} \sim 1$. 


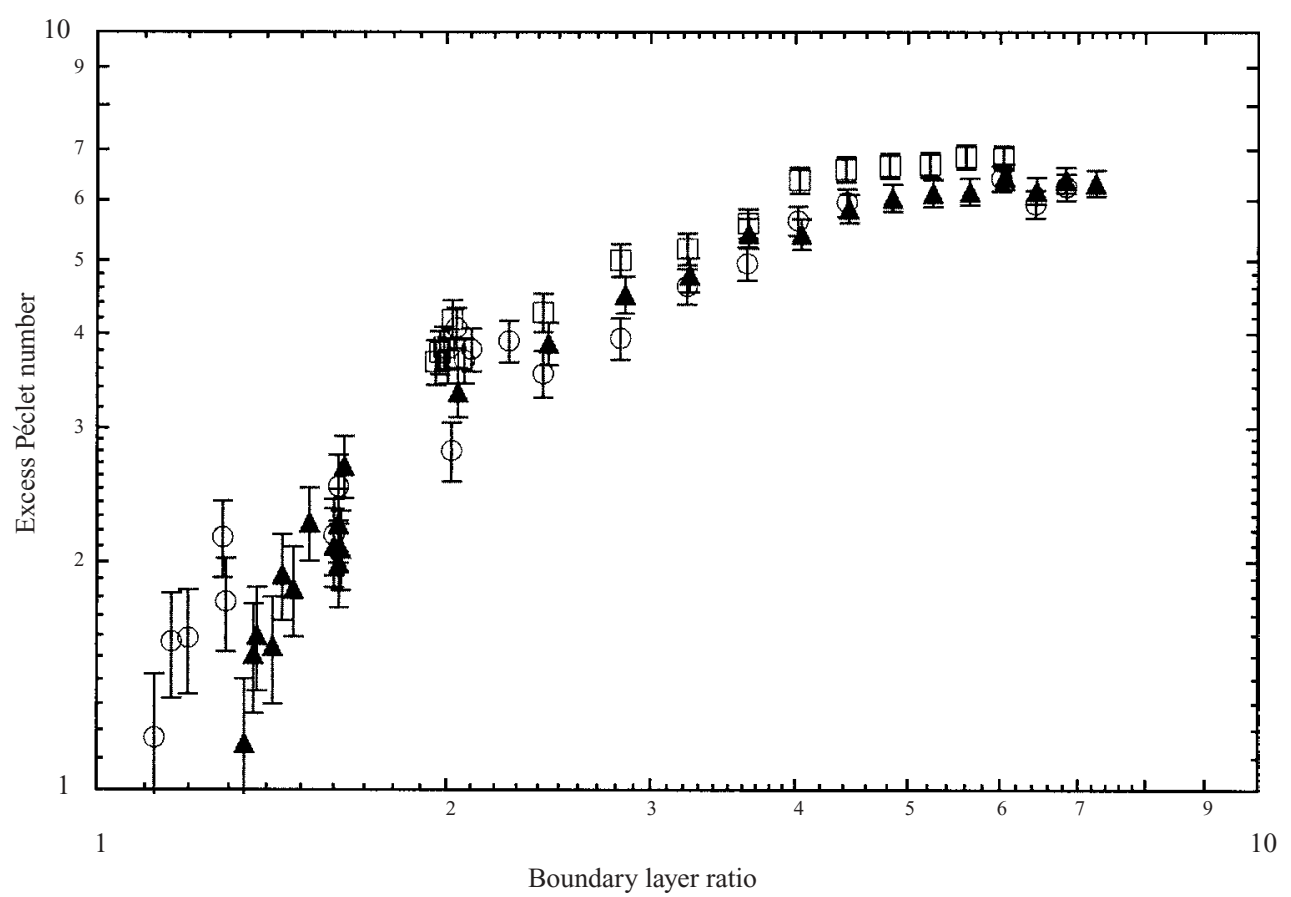

FIGURE 4. Variation of dimensionless excess heat transport in flow in a rotating differentially heated annulus with boundary layer parameter $\mathscr{P}$, covering the range within which regular baroclinic waves occur. Excess Péclet number is defined as the difference between measured total heat transport in laboratory experiments and the corresponding axisymmetric heat transport computed in numerical simulations at the same value of $\mathscr{P}$, normalized by the conductive heat transport consistent with the fluid properties and boundary conditions. Key is the same as for figure 1.

This is more clearly illustrated in figure 4, which shows the difference $P e_{x s}$ plotted against $\mathscr{P}$ over the complete range over which baroclinic waves occur. The error bars on each point here primarily indicate the uncertainty implied by the experimental precision in measuring $N u$. We see that, although the total heat transport $N u_{\text {total }}$ remains almost constant, both in the axisymmetric and regular baroclinic wave regimes (as previously noted e.g. by Hide \& Mason 1975), the contribution attributable to the baroclinic eddies increases directly as the contribution due to Ekman layer transports decreases over the range $\mathscr{P}>1$. In fact the eddy contribution appears to show some weak tendency towards saturation, and even a slight decrease at large values of $\mathscr{P}$, though this is not conclusive with the present dataset. In fact the effective eddy heat transport, measured as an excess in Nusselt or Péclet number, is also apparently relatively insensitive to the dominant wavenumber in the flow, though some small differences between wavenumbers are weakly evident, with the higher wavenumbers showing slightly larger values of $P e_{x s}$ for a given $\mathscr{P}$.

\subsection{Variations with thermal Rossby number}

Differences between the transports due to differing wavenumbers are somewhat clearer if the data are plotted with respect to the thermal Rossby number $\Theta$, rather than $\mathscr{P}$. Figure 5 shows the same values of $P e_{x s}$ plotted against $\Theta$, defined 


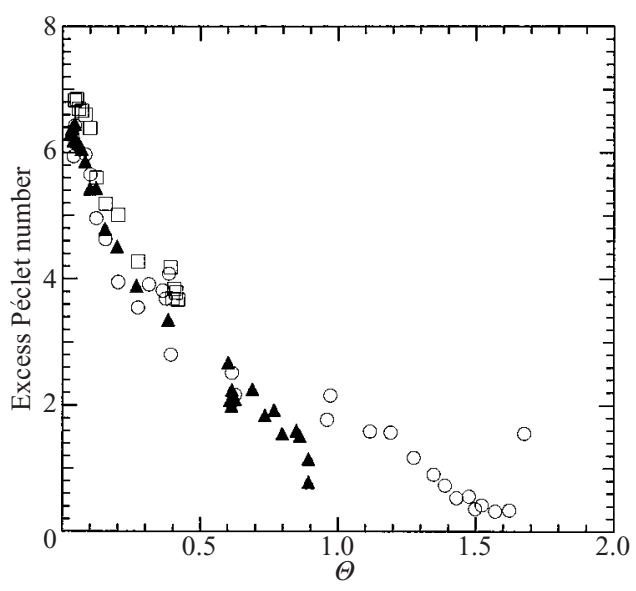

FIGURE 5. Variation of dimensionless excess heat transport $P e_{x s}$ in flow in a rotating differentially heated annulus with stability parameter or thermal Rossby number $\Theta$, covering the range within which regular baroclinic waves occur. Key is the same as for figure 1 .

as

$$
\Theta=\frac{g \alpha \Delta T}{\Omega^{2}(b-a)^{2}} .
$$

From this, it is clear that $P e_{x s}$ increases almost linearly as $\Theta$ decreases, but the line associated with each wavenumber intersects the axis at different values. From a least-squares linear fit of the data from each wavenumber against $\Theta$, we can find extrapolated values of $\Theta$ at which $P e_{x s}$ goes to zero, referred to hereafter as $\Theta_{c}$. Thus, $\Theta_{c}=1.55 \pm 0.05$ for $m=2, \Theta_{c}=1.04 \pm 0.05$ for $m=3$ and $\Theta_{c}=0.87 \pm 0.06$ for $m=4$.

It is interesting to note that these values of $\Theta_{c}$ correspond closely to the maximum values of $\Theta$ at which each wavenumber can be obtained at comparable values of Taylor number (e.g. see the regime diagram in Hignett et al. 1985). Thus, we may interpret $\Theta_{c}(m)$ as the critical value of $\Theta$ for the onset of fully developed baroclinic waves of wavenumber $m$.

\subsection{Variations with scaled 'supercriticality'}

Following on from this interpretation, we may then plot $P e_{x s}$ against the baroclinic 'supercriticality', $\Theta_{c}(m)-\Theta$, for each wavenumber, and the result is shown in figure 6 . Each wavenumber now follows a separate curve from the origin, with a somewhat different gradient in each case. However, the almost linear variation is now clear, though the total excursion in the present series of measurements does not exceed an excess Nusselt number $P e_{x s}$ of around 7-8. This is despite the observation that $m=2$ is observed throughout the range of $\Theta$ covered, whereas $m=4$ is only found for a relatively small range of $\Theta$.

Finally, we note that if the 'supercriticality' is scaled in each case by $\Theta_{c}(m)$ for each wavenumber set, then all the measurements can apparently be reduced to a single curve for 'normalized supercriticality' $\eta=1-\Theta / \Theta_{c}$. The result is illustrated in figure 7 , which clearly shows all three wavenumber curves collapsing onto a single relationship between $P e_{x s}$ and $\eta$ for small $\eta(\leqslant 0.4)$, though some divergence between wavenumbers is apparent for $\eta>0.5$. The overall impression, therefore, is that the total heat transport attributable to the presence of eddies depends rather 


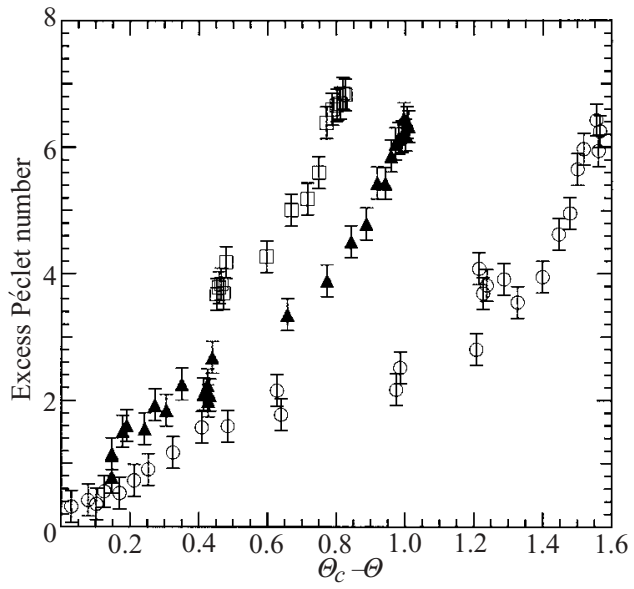

Figure 6. Variation of dimensionless excess heat transport $P e_{x s}$ in flow in a rotating differentially heated annulus, shown plotted with respect to a 'critical' stability parameter or thermal Rossby number $\Theta_{c}$ for each regular baroclinic wavenumber $m=2(\bigcirc), m=3(\triangle)$ and $m=4(\square)$.

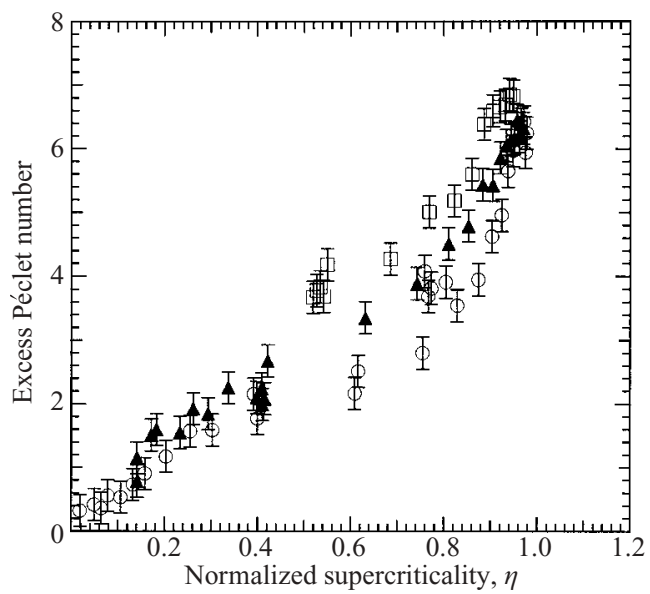

FiguRE 7. Variation of dimensionless excess heat transport in flow in a rotating differentially heated annulus, shown plotted with respect to a 'critical' stability parameter or thermal Rossby number $\Theta_{c}$, itself normalized by $\Theta_{c}$, for each regular baroclinic wavenumber $m=2(\bigcirc), m=3$ $(\triangle)$ and $m=4(\square)$.

simply (almost linearly) on supercriticality, based on a dimensionless parameter which relates directly to similar parameters (e.g. the Burger number) derived from linear and weakly nonlinear theories for baroclinic instability, provided $\eta$ is not too large. This will be discussed in more detail in the following section.

\section{Discussion}

The work presented in this paper has shown that, as the effect of background rotation increases, baroclinic waves carry an increasing proportion of the total heat 
transport in a rotating differentially heated annulus, while the direct transport by the axisymmetric boundary-layer circulation decreases. The result may lead (at least in some circumstances) to the total heat transport remaining almost independent of $\Omega$ over a substantial range of rotation. Whether this is necessarily the case is a matter for further investigation, though it would seem reasonable for the flow to try to maintain the maximum possible heat transport (essentially set by the strength of the sidewall thermal boundary layers - which is mainly dependent on the Rayleigh number, e.g. as $R a^{1 / 4}$ for laminar conditions) until circumstances (such as secondary instabilities) prevent the baroclinic waves from effecting the required transport (possibly suggesting a form of 'thermostatic adjustment', cf. Stone 1978).

\subsection{Axisymmetric and 'eddy' heat transports}

The approach adopted here, in utilizing a fully validated numerical model to compute the component of total heat transport due to axisymmetric motions, neatly sidesteps the (sometimes stormy) debate over how to separate 'eddy' and 'zonal mean' transports from the explicit calculation of variances and correlations. Provided we can trust the numerical model to compute accurately the total heat transport carried by the purely axisymmetric flow at the required combination of external parameters, and this has been verified to better than $\pm 2.5 \%$ in the actual axisymmetric regime where baroclinic waves do not occur (Read et al. 2000), then the difference between the measured heat transfer in the laboratory and the numerically determined axisymmetric transport must be attributable to the integrated effect of the presence of fully developed baroclinic waves. Such a result is independent of which coordinate surface is used to evaluate zonal averages, and must therefore in some sense be regarded as 'fundamental' to the flow.

In many discussions of this type of problem, it is frequently asserted that baroclinic eddies serve to transport heat horizontally to reduce the large-scale horizontal thermal gradient, and to transport heat upwards, which helps to release potential energy and also to enhance the mean static stability. Such mixing hypotheses are contained, for example, within closure theories such as those due to Green (1970), Stone (1972), Pfeffer \& Barcilon (1978). Given an ability to simulate the full flow numerically, as both an axisymmetric flow (with baroclinic instability suppressed) and a fully timedependent, baroclinically unstable flow in three dimensions, we can directly compare the effects of the development of baroclinic eddies from a comparison of simulations in two and three dimensions at the same point in parameter space.

Figure 8, for example, shows cross-sections of temperature from axisymmetric and fully three-dimensional simulations using the model described above at a resolution of $24 \times 24$ points in $r$ and $z$ and (in three dimensions) of 64 points in azimuth, for conditions within the regular steady $m=3$ wave regime in the thermal annulus, as discussed above. The three-dimensional run was initialized from the steady axisymmetric state illustrated in figure $8(a)$ and integrated for a further period to allow baroclinic waves to develop. The three-dimensional field in figure $8(b)$ corresponds to a zonal mean section after around $2000 \mathrm{~s}$ of simulated time, by which time the baroclinic instability has saturated at its fully developed (steady) amplitude. The resulting zonal mean fields are therefore representative of a new steady state of the system, and indistinguishable (in this case) from a space-time average. The difference plot in figure $8(c)$ shows the predominant change brought about by the development of the baroclinic eddies, in primarily reducing the horizontal thermal gradient in the approximately quasi-geostrophic interior, and hence the baroclinicity 

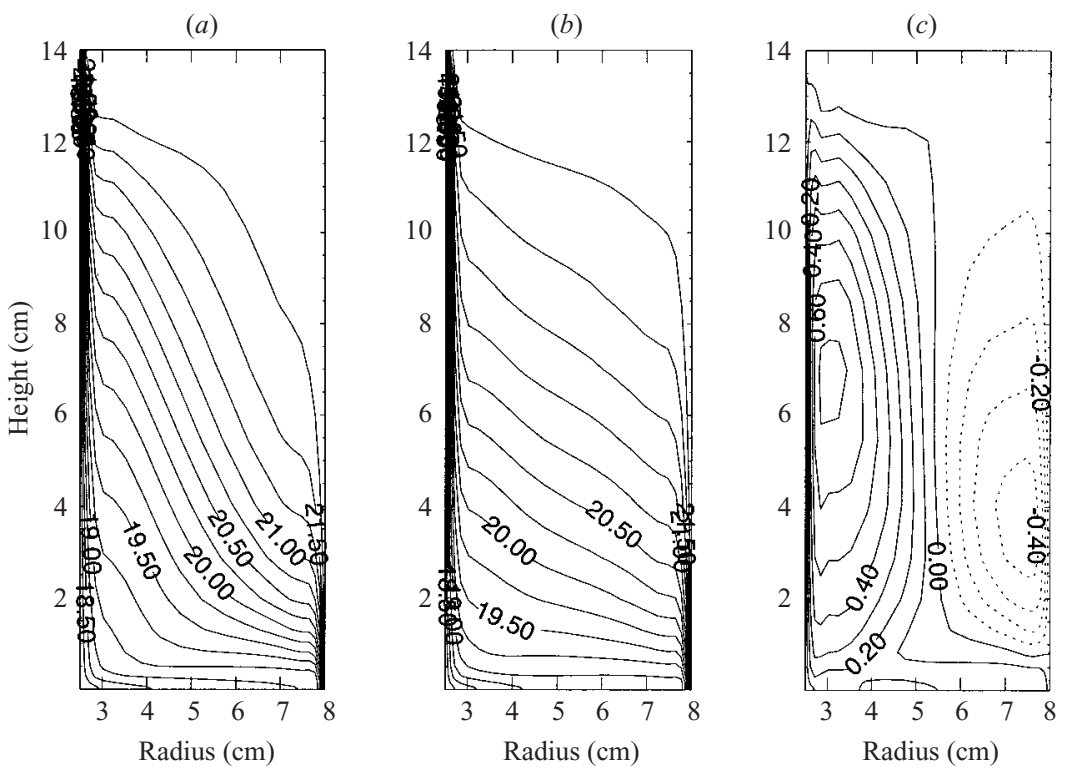

FiguRE 8. Temperature cross-sections in $(r, z)$ from numerical simulations of baroclinic flow in a differentially heated rotating annulus. $(a)$ The steady axisymmetric flow at $(\Theta, E)=$ $\left(0.619,5.41 \times 10^{-5}\right) ;(b)$ the corresponding zonal-mean temperature field from the threedimensional simulation, initialized from $(a)$ and integrated for $\sim 1500$ s until the dominant baroclinic waves $(m=3)$ have equilibrated. The difference field $T$ (three-dimensional) $T$ (two-dimensional) is shown in $(c)$.

of the flow. In this example, the modification to the overall static stability as a result of baroclinic instability seems relatively small. The equilibrated Nusselt number in this simulation is $\sim 10.9$ (cf. the measured value of $\sim 10.2$ ), although the numerical model at this comparatively modest resolution tends systematically to overestimate the Nusselt number by $\sim 5 \%$ (cf. Read et al. 2000).

It is noteworthy that figure $8(b)$ is not a simple rearrangement of figure $8(a)$, as would be expected if baroclinic transports were adiabatic. Such a conclusion is clearly apparent from figure $8(c)$, which does not average to zero and indicates a net heating of the fluid during the development of the three-dimensional flow. This is not unduly surprising in this case, since the diagnostics of the three-dimensional flow were computed around 1 hour later (in simulated time) than the axisymmetric flow used as its initial condition. During this time, enhanced baroclinic transports were able to accelerate the 'ventilation' of isothermal layers in the fluid in the thermal boundary layers adjacent to the sidewalls. The net effect of the baroclinic eddy transports is therefore not purely adiabatic when taken over a finite time interval. Such an effect is not generally considered explicitly in geophysical applications of eddy parametrizations, though, as in the present situation, may be far from negligible.

\subsection{Transport efficiency and parameterizations based on baroclinic instability theory}

From the variation of $P e_{x s}$ presented in $\S 4$, we have a measure of how the efficiency of baroclinic eddy heat transport is found to vary with external parameters throughout the quasi-regular wave regime of the thermal annulus. From figures 4-6 we see that $P e_{x s}$ generally increases with $\mathscr{P}$ and $\Theta_{c}-\Theta$, with a scaling which suggests the 
importance of baroclinic supercriticality $\Theta_{c}-\Theta$, and then apparently saturates at a roughly constant (or perhaps weakly decreasing) value at the highest values of $\Omega$.

As briefly discussed in the introduction, parametrizations of heat transport in ocean and atmosphere models have typically represented eddy fluxes as a diffusive transport as in equation (1.1). At least four main approaches to defining the eddy diffusion coefficient $\mathscr{K}$ have generally been proposed in the literature, all of which take the generic form (cf. Haine \& Marshall 1998)

$$
\mathscr{K} \simeq C_{E} v_{e d d y} \mu,
$$

where $v_{\text {eddy }}$ is a velocity characteristic of eddy motion, $\mu$ is a horizontal lengthscale (like a mixing length) and $C_{E}$ is a dimensionless constant. The differences between the main approaches lie in the way in which they apply results from various forms of linear baroclinic instability theory to determine values for $v_{e d d y}$ and $\mu$. The simplest approach (as discussed e.g. by Gent \& McWilliams 1990; Gent et al. 1995; Larichev $\&$ Held 1995) effectively uses uniquely specified scales for $L_{z o n e}, N$ and $|\partial \bar{b} / \partial y|$, leading to a constant coefficient $\mathscr{K}$. More recently, however, schemes involving an eddy diffusion coefficient which depends on the local properties of the mean flow have been advocated, as outlined below.

\subsubsection{Scheme A: the Green (1970) model}

The most common approach (used e.g. by Visbeck et al. 1997 and Treguier et al. 1997) follows the original suggestion of Green (1970) and sets the lengthscale $\mu$ equal to the width of the baroclinic zone $L_{z o n e}$, whilst taking the timescale $\mu / v_{e d d y}$ to equal the growth timescale $\tau_{\text {Eady }}$ for the most unstable modes in the Eady model of baroclinic instability $(\sim \sqrt{R i} / f$, where $R i$ is the Richardson number). As discussed e.g. by Haine \& Marshall (1998), this leads to the canonical form for $\mathscr{K}$

$$
\mathscr{K}=C_{G} \frac{L_{\text {zone }}^{2}}{N}\left|\frac{\partial \bar{b}}{\partial y}\right|,
$$

where $C_{G}$ is a constant with a value $\sim 0.015-0.025$ (cf. Haine \& Marshall 1998), with essentially no dependence on rotation rate $f$.

\subsubsection{Scheme B: the Stone (1972) model}

An alternative approach, noted by Haine \& Marshall (1998), invokes a slightly different set of assumptions concerning the length- and timescales in (5.1). Following Stone (1972), $\mu$ may be taken to equal the Rossby deformation radius, $L_{R}=N D / f$, and $v_{e d d y}$ is taken to be a 'typical' thermal wind scale $v_{e d d y} \sim|\partial \bar{b} / \partial y| D / f$. This leads to the alternative form of $\mathscr{K}$

$$
\mathscr{K}=C_{S} \frac{L_{\text {zone }}^{2}}{N}\left|\frac{\partial \bar{b}}{\partial y}\right| B u,
$$

where $C_{S}$ is a further dimensionless constant, whose value may range from around 0.42 (Haine \& Marshall 1998) to 0.86 (Stone 1972), and $B u$ is the Burger number $\left(N D / f L_{z o n e}\right)^{2}$. This would suggest a heat transport efficiency which decreases quite rapidly with $f$, especially when the baroclinic zone is much wider than the local deformation radius. 


\subsubsection{Scheme C: the Haine \& Marshall (1998) model}

Haine \& Marshall (1998) suggested a further variation on the above theme, defining $\mu$ to equal the width $L_{z o n e}$ of the baroclinic zone, and $v_{\text {eddy }}$ to be the thermal wind scale defined above. In the present context, this leads to the comparable canonical form

$$
\mathscr{K}=C_{e} \frac{L_{\text {zone }}^{2}}{N}\left|\frac{\partial \bar{b}}{\partial y}\right|(B u)^{1 / 2},
$$

with $C_{e} \sim 0.1$ according to Haine \& Marshall (1998) for their numerical experiments at $B u=0.063$.

\subsubsection{Scheme D: the Pfeffer \& Barcilon (1978) model}

All of the schemes outlined above would appear to suggest that heat transport by eddies should be either independent of background rotation (indicated by (5.2)) or more or less decreasing with $f\left((5.3)\right.$ and (5.4)) since $B u \sim \Theta / 4 \sim f^{-2}$. Whilst the lack of a dependence on $f$ in Scheme A might be compatible with the variation of $P e_{x s}$ in the rapidly rotating irregular baroclinic wave regime applicable for $\mathscr{P} \geqslant 5$, it is clearly not consistent with the measured dependence of $P e_{x s}$ within the regular wave regime for $1 \leqslant \mathscr{P}<5$.

As mentioned above, a further alternative approach which could be in qualitative agreement with our measurements of $P e_{x s}$ in the regular wave regime is suggested from the work of Pfeffer \& Barcilon (1978). They analysed the dependence of eddy heat flux in the weakly nonlinear Eady-type baroclinic instability theory of Drazin (1972), in which an unstable baroclinic wave grows to finite amplitude and equilibrates. The detailed variation of equilibrated heat flux leads to a complicated dependence on various parameters, including the wavenumber and viscous dissipation (measured by the Ekman number), but the overall conclusion of this analysis suggests that the eddy heat flux should scale in this regime with the square of the equilibrated amplitude of the unstable baroclinic wave, such that

$$
\overline{v^{\prime} T^{\prime}} \sim S\left(\frac{\partial \bar{T}}{\partial y}\right)^{2}\left|B u_{c}-B u\right|,
$$

where $B u_{c}$ is the critical value of $B u$ for the onset of linear baroclinic instability in the Eady model. In this expression, $S$ is a complicated function of the wavenumber, rotation rate, geometry and fluid properties, but the form of the function deduced by Pfeffer \& Barcilon (1978) suggests that $\overline{v^{\prime} T^{\prime}}$ will increase weakly with wavenumber $m$. In addition, $\partial \bar{T} / \partial y$ in the above expression strictly refers to the thermal gradient at marginal stability, and not the actual thermal gradient in the presence of the fully developed waves. By comparison with the other schemes discussed above, however, we can represent the predicted variation of eddy diffusivity in this model by

$$
\mathscr{K} \simeq C_{P B}^{\prime} \frac{L_{\text {zone }}^{2}}{N}\left|\frac{\partial \bar{b}}{\partial y}\right|\left|B u_{c}-B u\right|
$$

or

$$
\mathscr{K} \simeq C_{P B} \frac{L_{\text {zone }}^{2}}{N}\left|\frac{\partial \bar{b}}{\partial y}\right|\left|\Theta_{c}-\Theta\right|,
$$

where $C_{P B}$ and $C_{P B}^{\prime}$ are dimensionless constants, which may in turn depend on suitably non-dimensionalized wavenumbers and the Ekman number of the flow. 
Given the provisos mentioned above, therefore, we can see that the weakly nonlinear approach leads to an approximate expectation that baroclinic eddy heat transport will have a linear dependence on supercriticality $\left|\Theta_{c}-\Theta\right|$ and a quadratic dependence on the lateral thermal gradient in the zonal mean. From the analyses of Hignett et al. (1981) and Read (1986, 1992), however, the axisymmetric isotherm slope is expected to become large but only weakly increasing with $\Omega$ in the parameter regimes where baroclinic instability becomes prevalent. In contrast, laboratory observations of $\partial \bar{T} / \partial y$ in the regular wave regime (e.g. Pfeffer \& Barcilon 1978) suggest a weak inverse dependence of this quantity on $\Omega$. For the purposes of the present discussion, therefore, it may be appropriate to consider $\partial \bar{T} / \partial y$ to be roughly constant within the regular wave regime in the laboratory, in which case, the dependence indicated in (5.5) and (5.7) could be consistent with the measurements of $P e_{x s}$ presented above.

\subsection{TEM parametrizations in the rotating annulus}

As outlined in the introduction to this paper, an alternative view of the role of baroclinic eddy transports is suggested from the work of Gent \& McWilliams (1990), based on the transformed Eulerian mean (TEM) approximation to the Lagrangian mean formulation of wave-zonal flow interactions. According to this approach, the net transport of heat (and other tracers) is represented in the Eulerian zonal mean (along suitable coordinate surfaces, such as geopotential height or pressure) by an additional lateral and vertical component of velocity $\left(v_{*}, w_{*}\right)$, such that in the present context of cylindrical geometry

$$
\begin{aligned}
& v_{*}=\frac{1}{r} \frac{\partial}{\partial z}\left(\frac{\overline{v^{\prime} T^{\prime}}}{\partial \bar{T} / \partial z}\right), \\
& w_{*}=-\frac{1}{r} \frac{\partial}{\partial r}\left(\frac{\overline{v^{\prime} T^{\prime}}}{\partial \bar{T} / \partial z}\right) .
\end{aligned}
$$

This may be further represented as a meridional streamfunction $\chi_{*}$, given by

$$
\chi_{*}=\left(\frac{\overline{v^{\prime} T^{\prime}}}{\partial \bar{T} / \partial z}\right) .
$$

As an illustration, we compare in figure 9 the meridional streamfunctions $(a)$ $\chi_{a x}$ for the axisymmetric numerical simulation whose temperature field is shown in figure $8(a)$, and $(b)$ the streamfunction for the full transport circulation (including the zonal mean explicit meridional flow and the TEM circulation), defined as $\chi_{T}=\chi+\chi_{*}$, determined from the fully three-dimensional baroclinic wave simulation shown in figure $8(b)$. The difference streamfunction $\chi_{T}-\chi_{a x}$, representing the extra meridional transport attributable to the presence of the baroclinic eddies, is shown in figure $9(c)$, for comparison. Note that $\chi_{T}-\chi_{a x}$ is approximately the same as (though not strictly identical to) the TEM streamfunction $\chi_{*}$. From this example, we can see that the additional transport due to the presence of the baroclinic eddies, as represented in the TEM framework by the pattern of $\overline{v^{\prime} T^{\prime}} /(\partial \bar{T} / \partial z)$ in the equilibrated flow, leads to an additional overturning circulation in the interior of the annulus which will transport heat roughly downgradient with respect to the zonal mean temperature field. 
(a)

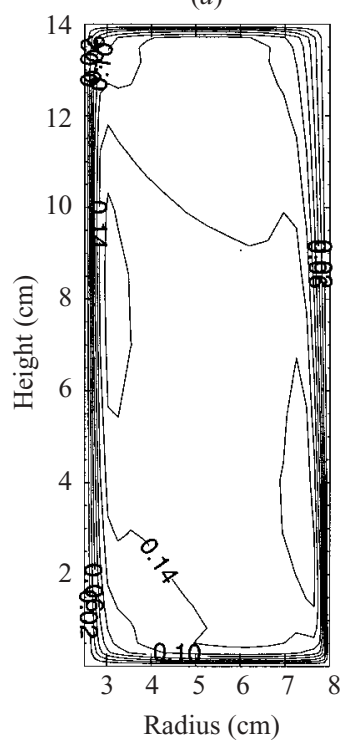

(b)

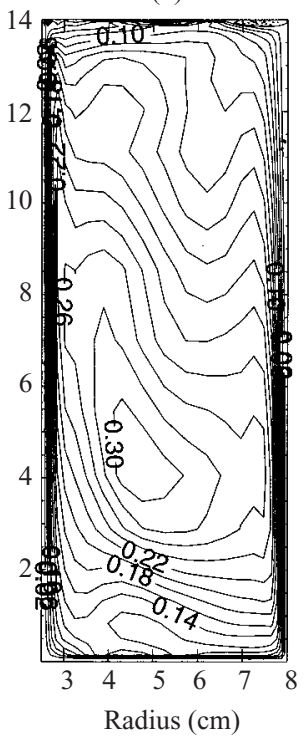

(c)

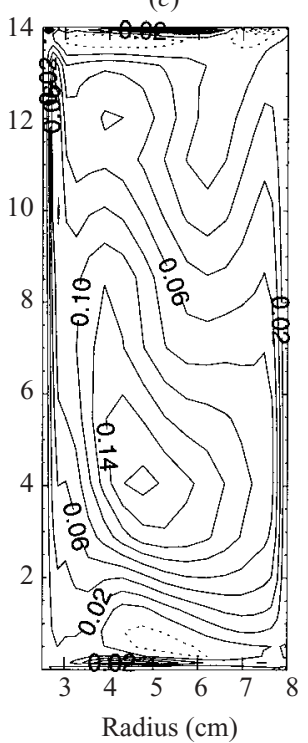

FiguRE 9. Cross-sections in $(r, z)$ of the meridional streamfunction $\chi$ from numerical simulations of baroclinic flow in a differentially heated rotating annulus. $(a) \chi_{a x}$ for the steady axisymmetric flow at $(\Theta, E)=\left(0.619,5.41 \times 10^{-5}\right) ;(b)$ the corresponding zonal-mean meridional streamfunction $\chi_{T}$ from the three-dimensional simulation, initialized from $(a)$ and integrated for $\sim 1500 \mathrm{~s}$ until the dominant baroclinic waves $(m=3)$ have equilibrated. The difference field $\chi_{T}-\chi_{a x}$ is shown in $(c)$.

The approach to parametrization of heat transport advocated by Gent \& McWilliams (1990), and being used increasingly in the oceanographic world, entails the replacement of $\overline{v^{\prime} T^{\prime}}$ by a function of the mean flow, such as the diffusive approximation in (1.1). As mentioned above, Gent \& McWilliams (1990) employed a form of (1.1) in which $\mathscr{K}$ was a specified constant, regardless of the form of the mean flow. In the present context, however, we follow e.g. Visbeck et al. (1997) in allowing for the possibility of a flow-dependent form for $\mathscr{K}$. The pattern of $\chi_{*}$ in the three-dimensional simulation discussed above is illustrated in figure $9(c)$, and would seem to suggest a correlation between $\chi_{*}$ and $\partial \bar{T} / \partial r$ within the flow, at least well outside boundary layers. This is more apparent if we directly plot values of (suitably scaled) eddy heat flux, computed in the three-dimensional simulation, against $\partial \bar{T} / \partial r$. Figure 10 shows an example of such a correlation, derived from the simulation shown in figures 8 and 9. For consistency with the previous discussion, we show the quantity $-\overline{v^{\prime} T^{\prime}}(\partial \bar{T} / \partial z)^{1 / 2} \sim-\overline{v^{\prime} T^{\prime}} N$ plotted against $\partial \bar{T} / \partial r$ at each model grid point throughout the meridional $(r, z)$-plane of the annulus lying outside the main boundary layers. From this plot, it is clear that the scaled heat flux and zonal mean temperature gradient are strongly anti-correlated, as suggested from all of the simple parametrizations discussed above. In the context of Scheme A, the fit shown in figure 10 is consistent with an implied value for $C_{G}$ of $0.019 \pm 0.001$, in good agreement with the range suggested above by Visbeck et al. (1997) and Haine \& Marshall (1998). If we regard Scheme $D$ as more appropriate in this case, then $C_{P B} \simeq 0.020$ if we take $\Theta_{c}=1.55$ as suggested above. The clear correlation between $-\overline{v^{\prime} T^{\prime}} N$ and $\partial \bar{T} / \partial r$ would seem to indicate that a Gent-McWilliams approach to parametrization in the thermally driven rotating annulus, but based on a 


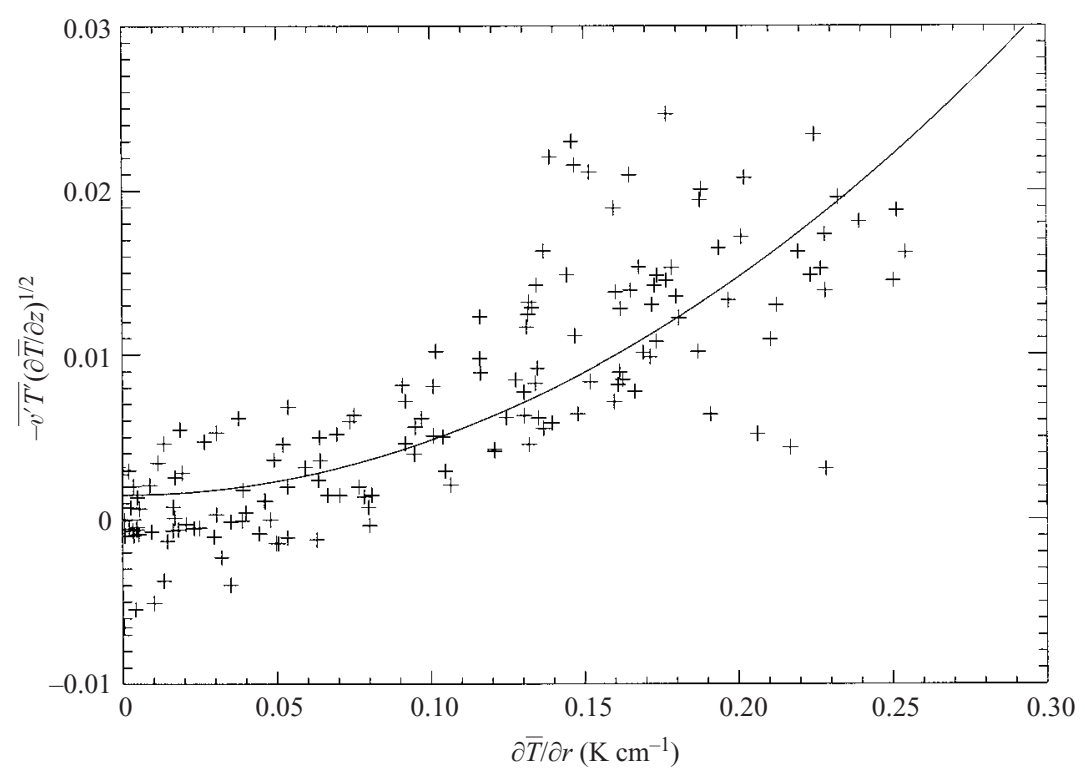

FIGURE 10. Correlation of $-\overline{v^{\prime} T^{\prime}}(\partial \bar{T} / \partial z)^{1 / 2}$ and $\partial \bar{T} / \partial r$ from the three-dimensional simulation illustrated in figures 8 and 9 at $(\Theta, E)=\left(0.619,5.41 \times 10^{-5}\right)$. Both quantities were computed at each grid point in the meridional plane and plotted for all points more than $0.5 \mathrm{~cm}$ away from each boundary (to exclude spurious correlations in boundary layers). The solid line shows the result of a least-squares fit to the data of a quadratic relationship of the form $-\overline{v^{\prime} T^{\prime}}(\partial \bar{T} / \partial z)^{1 / 2}=G(\partial \bar{T} / \partial r)^{2}+F$, where $G$ and $F$ are constants with values $G=0.33 \pm 0.02$ and $F=1.5 \pm 0.5 \times 10^{-3}$.

flow-dependent diffusive closure such as the schemes reviewed above, might well offer a reasonably accurate representation of the effects of heat transport by baroclinic waves in the laboratory.

The choice of a coefficient $\mathscr{K}$ in (1.1) defined for Scheme A above, together with a weak dependence of $(\partial \bar{T} / \partial r)$ on $\Omega$, for example, would be consistent in the present context with a roughly constant value of $P e_{x s}$ which was independent of $\Omega$. As discussed above, such a result does not seem to be consistent with our measurements of $P e_{x s}$ in the regular baroclinic wave regime in the laboratory which, in contrast, appear to be more consistent with the weakly nonlinear Scheme D. However, at higher values of $\Omega$ in the geostrophic turbulence regime, the measurements suggest that $P e_{x s}$ does not continue to increase without limit, but seems to saturate or even begin weakly to decay with $\Omega$. In this case, one might speculate that a simpler parametrization based on Scheme A might be applicable, though such a possibility should be investigated thoroughly and compared with measurements in an appropriate dynamical regime, ideally by directly implementing parametrizations in a suitable numerical model. From the present experiments, however, the measurements do not appear to be consistent with either Scheme B or C in any regime investigated so far, although measurements taken much further into the fully developed geostrophic turbulence regime (and hence over a wider range of $B u$ ) are really needed to confirm this.

\subsection{Concluding remarks}

In the present study, we have taken the first steps towards demonstrating a novel and insightful approach to the quantitative determination of the role of baroclinic eddies 
in transporting heat in a differentially heated rotating fluid in the laboratory. The combined approach, using both laboratory calorimetric measurements and numerical simulations, allows an unambiguous assessment of the heat transport attributable to baroclinic eddies, even when heat is being carried in the full system via a variety of differing mechanisms.

The results presented here, together with the discussion of the previous subsections, indicate that an approach to the parametrization of baroclinic eddy transports along the lines of that suggested by Gent \& McWilliams (1990) may offer significant promise. In future work, therefore, it will be desirable $(a)$ to investigate the robustness of the present results in other baroclinically unstable systems, including an exploration of flows which are much more strongly supercritical than covered in the present work, and $(b)$ to investigate more closely the extent to which a practical parametrization of $\chi_{*}$ based on zonal mean fields and knowledge of external parameters may be feasible and/or appropriate. With respect to $(a)$, further laboratory experiments covering a wider range of external parameters and/or boundary conditions, and for which accurate total heat transport measurements are obtainable, would be extremely useful. For $(b)$, it will be necessary to carry out further numerical modelling studies, both to diagnose the heat transport in other fully three-dimensional simulations and to implement and evaluate suitable parametrizations in an otherwise two-dimensional axisymmetric model. Such studies might also enable an evaluation of the extent to which this approach could be applied to a prediction of the transport of other quantities, such as potential vorticity, material tracers or angular momentum.

Finally, one might hope that the successes derived from applying this approach to the differentially heated annulus would be transferable to a range of other systems, including zonally symmetric or time-averaged models of planetary atmospheres or oceans, and in the design of various forms of rotating machinery for which a parametrization of non-axisymmetric transports could have significant practical value.

I am grateful to Dr P. Hignett and Mssrs R. M. Small and W. D. N. Jackson of the Geophysical Fluid Dynamics Laboratory at the UK Met. Office for their efforts in constructing the original apparatus and making the laboratory measurements used in this study. Thanks are also due to Dr W. G. Früh for help in recalibrating these earlier heat transport measurements, to Dr A. A. White and Mr N. P. J. Thomas for advice and assistance with the numerical model, and to Dr T. W. N. Haine for insightful discussions on the issues raised in this paper. I am grateful to the anonymous reviewers of this paper for further constructive comments and suggestions which significantly improved the discussion and presentation.

\section{REFERENCES}

Andrews, D. \& MCINTYRe, M. 1978 An exact theory for nonlinear waves on a lagrangian-mean flow. J. Fluid Mech. 89, 609-646.

Danabasoglu, G., McWilliams, J. C. \& Gent, P. 1994 The role of mesoscale tracer transports in the global ocean circulation. Science 264, 1123-1126.

Drazin, P. G. 1972 Nonlinear baroclinic instability of a continuous zonal flow of a viscous fluid. J. Fluid Mech. 55, 577-587.

Gent, P. \& McWilliams, J. 1990 Isopycnal mixing in ocean circulation models. J. Phys. Oceanogr. 20, $150-155$.

Gent, P., Willebrand, J., McDougall, T. \& McWilliams, J. 1995 Parametrizing eddy-induced tracer transports in ocean circulation models. J. Phys. Oceanogr. 25, 463-474. 
GreAtBATCH, R. J. 1998 Exploring the relationship between eddy-induced transport velocity, vertical momentum transfer, and the isopycnal flux of potential vorticity. J. Phys. Oceanogr. 28, 422432.

Green, J. S. A. 1970 Transfer properties of the large-scale eddies and the general circulation of the atmosphere. Q. J. R. Met. Soc. 96, 157-185.

Griffies, S. M. 1998 The Gent-McWilliams skew flux. J. Phys. Oceanogr. 28, 831-841.

Haine, T. W. N. \& Marshall, J. C. 1998 Gravitational, symmetric and baroclinic instability of the ocean mixed layer. J. Phys. Oceanogr. 28, 534-658.

Held, I. M. \& LARICHEv, V. D. 1996 A scaling theory for horizontally homogeneous baroclinically unstable flow on a beta plane. J. Atmos. Sci. 53, 946-952.

Hide, R. \& MASON, P. J. 1975 Sloping convection in a rotating fluid. Adv. Phys. 24, 47-100.

HignetT, P. 1982 A note on the heat transfer by the axisymmetric thermal convection in a rotating fluid annulus. Geophys. Astrophys. Fluid Dyn. 19, 293-299.

HignetT, P. 1985 Characteristics of amplitude vacillation in a differentially heated rotating fluid annulus. Geophys. Astrophys. Fluid Dyn. 31, 247-281.

Hignett, P., Ibbetson, A. \& Killworth, P. D. 1981 On rotating thermal convection driven by non-uniform heating from below. J. Fluid Mech. 109, 161-187.

Hignett, P., White, A. A., Carter, R. D., Jackson, W. D. N. \& Small, R. M. 1985 A comparison of laboratory measurements and numerical simulations of baroclinic wave flows in a rotating cylindrical annulus. Q. J. R. Met. Soc. 111, 131-154.

Killworth, P. D. 1997 On the parametrization of eddy transfer. part i: Theory. J. Mar. Res. 55, 1171-1197.

Large, W., McWilliams, J. C. \& Doney, S. 1994 Oceanic vertical mixing: A review and a model with a nonlocal boundary layer parametrization. Rev. Geophys. 32, 336-403.

Larichev, V. D. \& Held, I. M. 1995 Eddy amplitudes and fluxes in a homogeneous model of fully developed baroclinic instability. J. Phys. Oceanogr. 25, 2285-2297.

Marshall, D. P., Williams, R. G. \& Lee, M.-M. 1999 On the relation between eddy-induced transport and isopycnic gradients of potential vorticity. J. Phys. Oceanogr. 29, 1571-1578.

Owen, J. M. \& Rogers, R. H. 1995 Flow and Heat Transfer in Rotating Disc Systems, Vol. 2: Rotating Cavities. Taunton, UK: Research Studies Press, John Wiley.

Pavan, V. \& Held, I. M. 1996 The diffusive approximation for eddy fluxes in baroclinically unstable zonal jets. J. Atmos. Sci. 53, 1262-1272.

Pfeffer, R. L. \& BARCilon, A. 1978 Determination of eddy fluxes of heat and eddy temperature variances using weakly nonlinear theory. J. Atmos. Sci. 35, 2099-2110.

Pfeffer, R. L., BuzYnA, G. \& Kung, R. 1980 Relationships between eddy fluxes of heat, eddy temperature variances and basic-state temperature parameters in thermally driven rotating fluids. J. Atmos. Sci. 37, 2577-2599.

Plumb, R. \& Mahlman, J. 1987 The zonally-averaged transport characteristics of the GFDL general circualtion/transport model. J. Atmos. Sci. 44, 298-327.

READ, P. L. 1986 Regimes of axisymmetric flow in an internally heated rotating fluid. J. Fluid Mech. 168, 255-289.

READ, P. L. 1992 Rotating annulus flows and baroclinic waves. In Rotating Fluids in Geophysical and Industrial Applications (ed. E. Hopfinger), pp. 185-214. Springer.

Read, P. L., Thomas, N. P. J. \& Risch, S. H. 2000 An evaluation of Eulerian and semi-Lagrangian advection schemes in simulations of rotating, stratified flows in the laboratory. part $\mathrm{i}$ : axisymmetric flow. Mon. Wea. Rev. 128, 2835-2852.

REDI, M. 1982 Oceanic isopycnal mixing by coordinate rotation. J. Phys. Oceanogr. 12, 1154-1157.

Robitaille, D. Y. \& Weaver, A. J. 1995 Validation of sub-grid scale mixing schemes using CFCs in a global ocean model. Geophys. Res. Lett. 22, 2917-2920.

Smith, R. D., Maltrud, M. E., Bryan, F. O. \& Hecht, M. W. 2000 Numerical simulation of the North Atlantic at $1 / 10^{\circ}$ r. J. Phys. Oceanogr. 30, 1532-1561.

STONE, P. 1972 A simplified radiative-dynamical model for the static stability of rotating atmospheres. J. Atmos. Sci. 29, 405-418.

Stone, P. 1978 Baroclinic adjustment. J. Atmos. Sci. 35, 561-571.

TAYLOR, K. E. 1980 The roles of mean meridional motions and large-scale eddies in zonally averaged circulations. J. Atmos. Sci. 37, 1-19. 
Treguier, A. M., Held, I. M. \& Larichev, V. D. 1997 Parametrization of quasigeostrophic eddies in primitive equation ocean models. J. Phys. Oceanogr. 27, 567-580.

Visbeck, M., Marshall, J., Haine, T. \& Spall, M. 1997 Specification of eddy transfer coefficients in coarse-resolution ocean circulation models. J. Phys. Oceanogr. 27, 381-402.

Visbeck, M. J., Marshall, J. C. \& Jones, H. 1996 On the dynamics of convective "chimneys" in the ocean. J. Phys. Oceanogr. 26, 1721-1734.

WRIGHT, D. K. 1997 A new eddy mixing parametrization and ocean general circulation model. Int. WOCE News 26, 27-29. 